\begin{tabular}{|l|l|l|l|l|l|}
\hline \multirow{2}{*}{$\mathcal{C}$} & J O O R & \multicolumn{1}{|c|}{$\mathbf{1 2 2 2 3}$} & \multirow{2}{*}{ WILEY } & Dispatch: 13.8.14 & CE: Vijay T. \\
\cline { 2 - 3 } \cline { 5 - 6 } & Journal Code & Manuscript No. & & No. of pages: 6 & PE: Mohanapriya \\
\hline
\end{tabular}

\title{
A sleep bruxism detection system based on sensors in a splint - pilot clinical data
}

\author{
P. MCAUlifFE*, J. H. KIM ${ }^{\dagger}$, D. DiAMOND ${ }^{\dagger}$, K. T. LAU ${ }^{\dagger}$ \& B. C. O’CONNELL* *Department \\ of Restorative Dentistry o Periodontology, Trinity College Dublin, Dublin Dental University Hospital, Dublin, and National Centre For \\ I Sensor Research, CLARITY - Centre for Sensor Web Technologies, Dublin City University, Dublin, Ireland
}

\begin{abstract}
SUMMARY It is difficult in a dental setting to accurately diagnose sleep bruxism and to objectively assess the severity, frequency or natural history of the condition in an individual patient. Yet this information is essential for the management of sleep bruxism and to plan appropriate dental treatment. The objective of this study was to clinically test a device that could be used to record bruxism events in a home environment. Pressure sensors were developed for use under the surface of an occlusal splint, and circuitry was designed to facilitate the recording and wireless transmission of the pressure sensor signal to a computer. Controlled mandibular movements were carried out in vivo to simulate bruxism and non-bruxism patterns. These patterns of force application were graphically presented to
\end{abstract}

two examiners who were asked to identify the type of activity represented by the force curves. Examiners were largely able to distinguish bruxism from non-bruxism activity; the sensitivity ranged from $80 \%$ to $100 \%$ and the specificity from $75 \%$ to $100 \%$. Using sensors in an occlusal splint, it is possible to recognise the typical tooth contact patterns seen in sleep bruxism. Such a device may be useful for monitoring sleep bruxism over an extended period at home.

KEYWORDS: sleep bruxism, tooth wear, occlusal splints, bite force, remote sensing technologies, stomatognathic diseases

Accepted for publication 30 July 2014

\section{Background}

Sleep bruxism is a common chronic condition affecting approximately $8 \%$ of the population (1-3). If untreated or poorly managed, bruxism can cause considerable damage to the teeth that can be very difficult to repair. Retrospective studies have shown that veneers and crowns fail faster in an untreated bruxer compared to treated bruxers or non-bruxers. A lack of scientific understanding and cost effective, validated clinical tools make it difficult to objectively diagnose and monitor bruxism $(4,5)$. Clinical parameters such as tooth surface loss $(6)$, facial pain $(7,8)$ and selfreport (9) have been found to be poorly predictive of the real presence of bruxism, its temporal profile or its severity. The commonest treatment currently provided to patients who suffer from bruxism is an occlusal splint (or 'night guard') $(10,11)$. Splints do not stop bruxism activity, but at least temporarily reduce its intensity and protect the teeth from the effects of grinding forces $(12,13)$. The mechanism of action of splints remains unclear, and there are no specific clinical guidelines for several aspects of their usage (14-16). There is considerable heterogeneity of clinical opinion about the appropriate duration of use, the basis for discontinuation, the most effective design and the optimum materials (17). These issues need to be addressed with clinical studies to optimise treatment for patients with bruxism.

While there is little or no data describing patient satisfaction or compliance with splint use, anecdotally most dentists report that both satisfaction and 
compliance are low. Despite the potential benefits of an occlusal splint, frequency of usage is thought to decline over time, with comfort issues and doubt about the ongoing necessity of use cited as the most common reasons. This has major long-term clinical and health economic implications, and so, there is a need for tools that help to monitor and reinforce patient compliance.

This study describes the clinical testing of a force sensor-based bruxism detection system, and its performance in detecting simulated biting patterns consistently seen in bruxism patients.

\section{Materials and methods}

Details of the design and assembly of the sensor-containing splint have been published (18). The force sensor was placed within a clear acrylic splint, with the sensor material buried approximately $1 \mathrm{~mm}$ below the occlusal surface in the pre-molar/first molar region. The splint was fitted in the mouth of one subject. Wireless communication was established between the splint and a receiver unit connected by USB to a laptop computer. Ten stereotyped bruxism and ten non-bruxism movements were simulated. Five of the bruxism movements were intended to replicate the biting patterns seen in phasic bruxism, and five more were intended to replicate tonic bruxism movements or static clenching. The force of the bites was at the maximum voluntary contraction of the subject and all movements involved a forceful vertical closure of the jaw. The biting patterns are summarised in Table 1 . The ten non-bruxism actions included five swallowing movements and five of random non-functional oromandibular movements, such as talking, lip smacking and tooth tapping.

Table 1. Biting patterns used with the sensor-containing occlu2 sal splint to simulate phasic bruxism and clenching events

\begin{tabular}{ll}
\hline Biting pattern & \\
\hline Phasic & Tonic \\
\hline $5 \mathrm{~s}$ lead in & $5 \mathrm{~s}$ lead in \\
Bite for $2-3 \mathrm{~s}$ & Bite for $5-10 \mathrm{~s}$ \\
Release for $2 \mathrm{~s}$ & Release \\
Bite for $2-3 \mathrm{~s}$ & \\
Release for $2 \mathrm{~s}$ & \\
Bite for $2-3 \mathrm{~s}$ & \\
Release for $2 \mathrm{~s}$ & \\
\hline
\end{tabular}

The signal output from all movements was recorded as a text file (Microsoft Wordpad) and transported into a spreadsheet (Microsoft Excel) where it was presented as a line graph. The $x$-axis of the line graph represented time and the $y$-axis the A/D converted value of the resistance change of the sensor. As force was increased, the A/D converted value was decreased, meaning that the graph seemed to show the inverse of the force input pattern.

The twenty unlabelled graphs were printed on A4 paper; a random sequence programme was used to generate a random sequence of the graphs. Two sets of the graphs were assembled into a booklet. The two examiners were shown the primary characteristics of each stereotyped movement pattern. In each of these graphs, the critical elements in discerning the type of pattern depicted are the number of force inputs, the number of phases or repetitions, the duration of the pressure and likely variations or artefacts that might occur. It was also explained where possible similarities existed that might lead to confusion.

Once the principal investigator was satisfied that the examiners understood the relevant signal characteristics, they were then presented with the test booklet and asked to categorise each of the twenty graphs as bruxism or non-bruxism. This exercise was repeated with the graphs in a different random order 2 weeks later (Fig. 1).

\section{Results and discussion}

Examples of the force output graphs for phasic bruxism, clenching and swallowing are shown in Fig. 2-4. These represent the force profile detected by the sensors during each of these jaw movements. The movements during phasic bruxism were stereotyped to replicate the temporal patterns of mandibular muscle activity normally seen during RMMA episodes, as defined by the bruxism research diagnostic criteria of Lavigne et al. (19).

The breakdown of correct and incorrect identification of the signals, and the calculated sensitivity and specificity are shown in Tables 2 and 3. The dental examiners in this study had significant prior knowledge and understanding of the clinical and physiological basis for bruxism, although they had no prior experience in viewing or scoring bruxism on sleep study traces. While identification of these test signals was reasonably robust, there were challenges in 

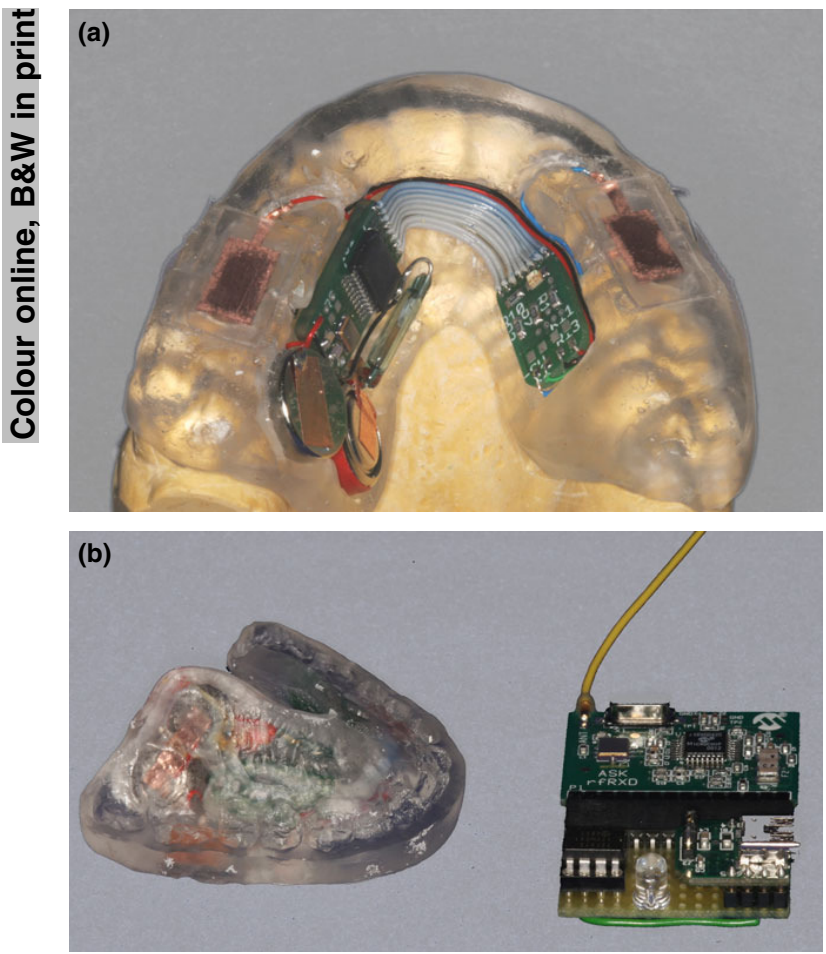

Fig. 1. (a) First prototype splint with electronics exposed before hermetic sealing (b) splint containing sensors and wireless receiver circuit.

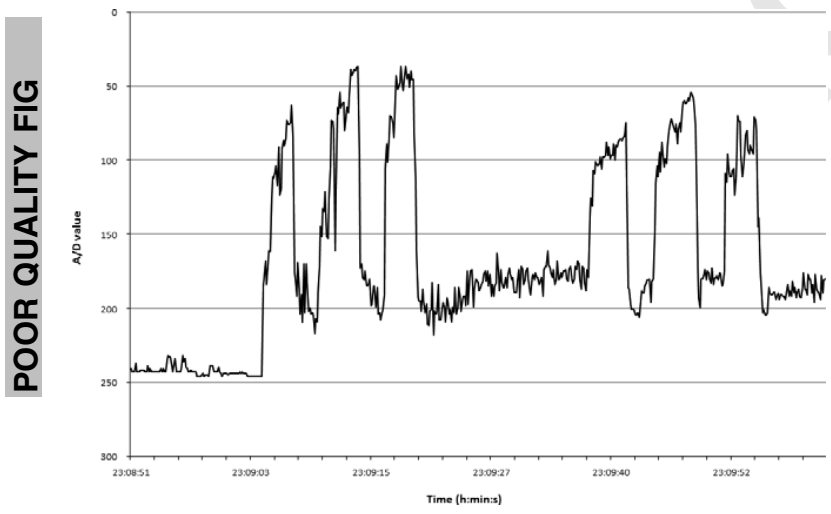

6 Fig. 2. In vivo response of wireless sensor-containing occlusal splint to forces simulating phasic bruxism, as described in Table 1 . The $x$-axis shows the time line of the recording, and the $y$-axis is the analogue-to-digital value of the device output, which represents the resistance of the sensor.

immediately distinguishing signals that appeared atypical in temporal pattern or where the amplitude of force detected seemed low. Hence, using the manual analysis of signals described, some intra- and interexaminer variation was seen. Despite the randomisation

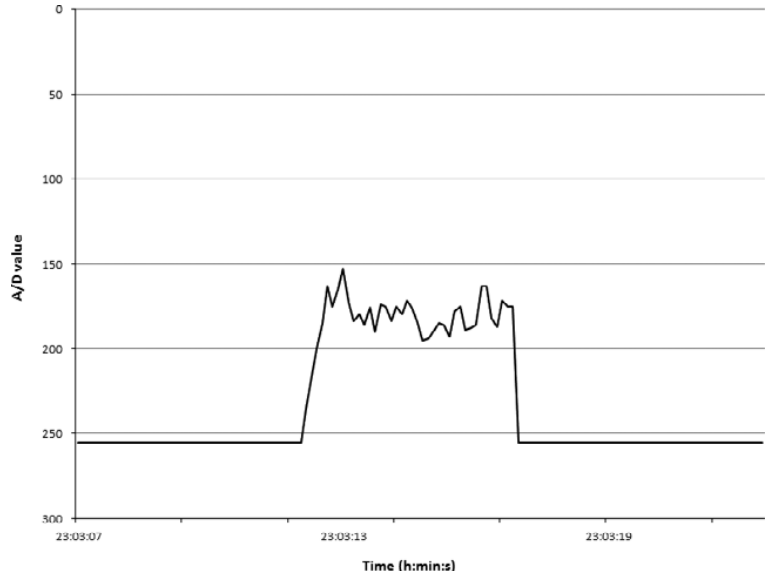

Fig. 3. In vivo response of wireless sensor-containing occlusal 4 splint to forces simulating tonic clenching, as described in Table 1 . The $x$-axis shows the time line of the recording, and the $y$-axis is the analogue-to-digital value of the device output, which represents the resistance of the sensor.
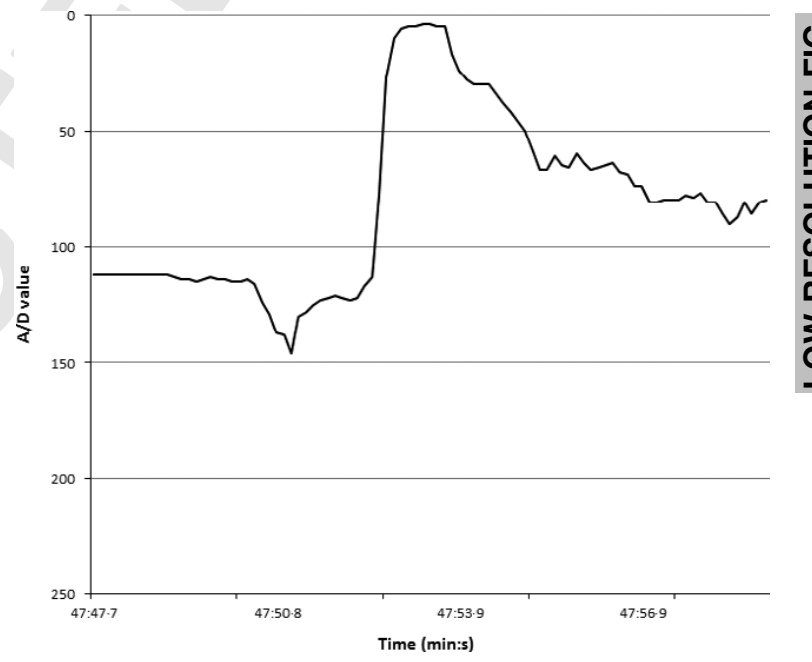

Fig. 4. In vivo response of wireless sensor-containing occlusal $\mathbf{5}$ splint to forces simulating swallowing. The $x$-axis shows the time line of the recording, and the $y$-axis is the analogue-to-digital value of the device output, which represents the resistance of the sensor.

of the sequence of unlabelled force graphs, it is difficult to completely eliminate bias or error as the examiners may have guessed that the sample contained a number of bruxing and non-bruxing events.

The data set recorded in this study was small and was intended as early proof of concept of a prototype device. Manual, visual assessment was therefore 
Table 2. Identification of bruxism and non-bruxism events by Examiner 1. The 95\% Confidence Limits for true sensitivity were $[62 \cdot 5,97 \cdot 5]$. The $95 \%$ Confidence Limits for true specificity were $[62 \cdot 5,97 \cdot 5]$

\begin{tabular}{llc}
\hline & True status & \\
\cline { 2 - 3 } Examiner 1 & Positive & Negative \\
\hline Examiner's estimate & 16 & 4 \\
Positive & 4 & 16 \\
Negative & $80 \%$ & \\
Sensitivity & & $80 \%$ \\
Specificity & & \\
\hline
\end{tabular}

Table 3. Identification of bruxism and non-bruxism events by Examiner 2. The $95 \%$ Confidence Limits for true sensitivity were $[100,100]$. The $95 \%$ Confidence Limits for true specificity were $[56,94]$

\begin{tabular}{lcc}
\hline & \multicolumn{2}{l}{ True status } \\
\cline { 2 - 3 } Examiner 2 & Positive & Negative \\
\hline Examiner's estimate & 20 & \\
Positive & 0 & 5 \\
Negative & $100 \%$ & 15 \\
Sensitivity & & $75 \%$ \\
Specificity & & \\
\hline
\end{tabular}

considered appropriate at this early stage. For data sets gathered during overnight recordings in longterm clinical studies, manual scoring would be unfeasible. A broader sample of overnight recordings from a large number of patients would be required to devise algorithms that were adequately robust to allow automated signal analysis. In constructing a force-based algorithm to allow more automated analysis of RMMA activity, it is necessary to initially characterise the relevant signals in detail and to ensure that the force detection system is capable of recording oro-mandibular activity with the required clarity.

Previous authors have used force detection systems embedded in mouthguards, although these studies had different objectives. Takeuchi et al. (20) examined the correlation between occlusal pressure signals and EMG activity by observing stereotyped movements of varying durations and patterns. Detailed analysis of the temporal relationship between EMG and pressure sensors showed that the latter provided excellent data on grinding movements, surpassing the signal quality of EMG. EMG had a clearer signal for clenching movements, however. This was related to the characteristics of the polymer sensor used, it being more suitable to the detection of dynamic on-off forces than static sustained clenches. The sensors used in the current study had no such limitations and were capable of detecting clenching more reliably than any other movement. Baba et al. (21) used a device similar to Takeuchi's in order to make overnight recordings in the ambulatory environment and establish that bruxism can be recorded successfully at home an occlusal force device. Watanabe et al. (22) extended the monitoring time to 3 weeks and the sample size to 12 bruxers; the purpose was to assess the presence of a correlation between certain psychological parameters and bruxism activity.

There were a number of technical features that the device used in these previous studies had in common. The devices used a digital sensing approach, where the number of events occurring over a pre-defined threshold value was counted. Hence, it would be difficult to characterise the force profile of typical types of bruxism activity or other common functional/parafunctional movements seen during sleep. The splint used in the present study contained sensors that could be customised according to the expected force profile and dynamic range. Most of the previously described devices had wires protruding from the mouth, a factor which could affect sleeping patterns and limit their use. Similarly, EMG-based detection of bruxism at home has been shown to be feasible, although the same issues with wired devices apply (23-25). By contrast, the hard PMMA device in the present study was wireless, albeit with a slightly increased bulk to accommodate the power source.

Nishigawa et al. (26) measured the bite force of 15 subjects using a slightly different technical approach. They used strain-gauge transducers incorporated in a simple acrylic bite-guard/splint that was wired to an external receiver. The results from this testing system were unique, in that they attempted to quantify exact mechanical bite forces of the subjects during specific bruxism episodes. The average force seen was $220 \mathrm{~N}$ with a maximum force of $896 \mathrm{~N}$ in one subject.

The focus of previous bruxism detection devices was on the analysis of simplified force signals to gather summary data on the frequency of bruxism events. The objective of this pilot study was to design and test a device that had the capacity to diagnose and analyse bruxism patterns in greater detail. 
Improved ability to distinguish different types of movement and their temporal characteristics offers three potential benefits. Firstly, it allows more concrete confirmation that the patterns of bruxism - as set out in the research diagnostic criteria (19) - are present in putative bruxism events and theoretically reduces the likelihood of artefacts and errors. Currently, bruxism movements are categorised as such primarily on the basis of their duration and pattern of EMG activity. Automated categorisation of these signals with some currently available sleep study devices may overestimate the severity of bruxism. The inclusion of a very detailed force signature in the analysis of these signals might improve sensitivity and specificity. Secondly, the use of a calibrated analogue sensor facilitates estimation of forces encountered during bruxism. This is potentially relevant clinically in treatment planning, particularly if the location and magnitude of the force could be identified. Finally, more detailed analysis of bruxism overnight in the ambient setting will provide valuable information on the true nature of the contacts involved and the variations seen within and between individuals.

\section{Disclosure}

This study was approved by the Health Sciences Faculty Research Ethics Committee, Trinity College. Funding was provided by Enterprise Ireland Proof of Concept Grant. The authors have no conflict of interests to disclose.

\section{References}

1. Lavigne GJ, Montplaisir JY. Restless legs syndrome and sleep bruxism: prevalence and association among Canadians. Sleep. 1994;17:739-743.

2. Maluly M, Andersen ML, Dal-Fabbro C, Garbuio S, Bittencourt L, de Siqueira JT et al. Polysomnographic study of the prevalence of sleep bruxism in a population sample. J Dent Res. 2013;92(7 Suppl):97S-103S.

3. Ohayon MM, Li KK, Guilleminault C. Risk factors for sleep bruxism in the general population. Chest. 2001;119:53-61.

4. Kato T, Yamaguchi T, Okura K, Abe S, Lavigne GJ. Sleep less and bite more: sleep disorders associated with occlusal loads during sleep. J Prosthodont Res. 2013;57:69-81.

5. Koyano K, Tsukiyama Y, Ichiki R, Kuwata T. Assessment of bruxism in the clinic. J Oral Rehabil. 2008;35:495-508.

6. Abe S, Yamaguchi $\mathrm{T}$, Rompre $\mathrm{PH}$, De Grandmont $\mathrm{P}$, Chen YJ, Lavigne GJ. Tooth wear in young subjects: a discrimina- tor between sleep bruxers and controls? Int J Prosthodont. 2009;22:342-350.

7. Rompre PH, Daigle-Landry D, Guitard F, Montplaisir JY, Lavigne GJ. Identification of a sleep bruxism subgroup with a higher risk of pain. J Dent Res. 2007;86:837-842.

8. Raphael KG, Sirois DA, Janal MN, Wigren PE, Dubrovsky B, Nemelivsky LV et al. Sleep bruxism and myofascial temporomandibular disorders: a laboratory-based polysomnographic investigation. J Am Dent Assoc. 2012;143:1223-1231.

9. Lavigne GJ, Khoury S, Abe S, Yamaguchi T, Raphael K. Bruxism physiology and pathology: an overview for clinicians. J Oral Rehabil. 2008;35:476-494.

10. Ommerborn MA, Taghavi J, Singh P, Handschel J, Depprich RA, Raab WH. Therapies most frequently used for the management of bruxism by a sample of German dentists. J Prosthet Dent. 2011;105:194-202.

11. Pierce CJ, Weyant RJ, Block HM, Nemir DC. Dental splint prescription patterns: a survey. J Am Dent Assoc. 1995; 126:248-254.

12. Dube C, Rompre PH, Manzini C, Guitard F, de Grandmont P, Lavigne GJ. Quantitative polygraphic controlled study on efficacy and safety of oral splint devices in tooth-grinding subjects. J Dent Res. 2004;83:398-403.

13. van der Zaag J, Lobbezoo F, Wicks DJ, Visscher CM, Hamburger HL, Naeije M. Controlled assessment of the efficacy of occlusal stabilization splints on sleep bruxism. J Orofac Pain. 2005;19:151-158.

14. Klasser GD, Greene CS, Lavigne GJ. Oral appliances and the management of sleep bruxism in adults: a century of clinical applications and search for mechanisms. Int J Prosthodont. 2010;23:453-462.

15. Lobbezoo F, van der Zaag J, van Selms MK, Hamburger HL, Naeije M. Principles for the management of bruxism. J Oral Rehabil. 2008;35:509-523.

16. Takahashi H, Masaki C, Makino M, Yoshida M, Mukaibo T, Kondo $\mathrm{Y}$ et al. Management of sleep-time masticatory muscle activity using stabilisation splints affects psychological stress. J Oral Rehabil. 2013;40:892-899.

17. Okeson JP. The effects of hard and soft occlusal splints on nocturnal bruxism. J Am Dent Assoc. 1987;1 14:788-791.

18. Kim JH, McAuliffe P, O'Connell B, Diamond D, Lau KT. Development of wireless bruxism monitoring device based on pressure-sensitive polymer composite. Sens Actuators, A. 2010;163:486-492.

19. Lavigne GJ, Rompre PH, Montplaisir JY. Sleep bruxism: validity of clinical research diagnostic criteria in a controlled polysomnographic study. J Dent Res. 1996;75:546-552.

20. Takeuchi H, Ikeda T, Clark GT. A piezoelectric film-based intrasplint detection method for bruxism. J Prosthet Dent. 2001;86:195-202.

21. Baba K, Clark GT, Watanabe T, Ohyama T. Bruxism force detection by a piezoelectric film-based recording device in sleeping humans. J Orofac Pain. 2003;17:58-64.

22. Watanabe T, Ichikawa K, Clark GT. Bruxism levels and daily behaviors: 3 weeks of measurement and correlation. J Orofac Pain. 2003;17:65-73. 
23. Haketa T, Baba K, Akishige S, Fueki K, Kino K, Ohyama T. Utility and validity of a new EMG-based bruxism detection system. Int J Prosthodont. 2003; 16:422-428.

24. Inano S, Mizumori $\mathrm{T}$, Kobayashi $\mathrm{Y}$, Sumiya M, Yatani $\mathrm{H}$. Identification of sleep bruxism with an ambulatory wireless recording system. Int J Prosthodont. 2013;26:527-535.

25. Mizumori T, Sumiya M, Kobayashi Y, Inano S, Yatani H. Prediction of sleep bruxism events by increased heart rate. Int J Prosthodont. 2013;26:239-243.
26. Nishigawa K, Kondo K, Takeuchi H, Clark GT. Contingent electrical lip stimulation for sleep bruxism: a pilot study. $\mathrm{J}$ Prosthet Dent. 2003;89:412-417.

Correspondence: Brian C. O'Connell, Department of Restorative Dentistry \& Periodontology, Dublin Dental University Hospital, Trinity College, Dublin 2, Ireland.

E-mail: brian.oconnell@dental.tcd.ie 


\section{Author Query Form}

Journal: JOOR

Article: $\quad 12223$

Dear Author,

During the copy-editing of your paper, the following queries arose. Please respond to these by marking up your proofs with the necessary changes/additions. Please write your answers on the query sheet if there is insufficient space on the page proofs. Please write clearly and follow the conventions shown on the attached corrections sheet. If returning the proof by fax do not write too close to the paper's edge. Please remember that illegible mark-ups may delay publication.

Many thanks for your assistance.

\begin{tabular}{|l|l|l|}
\hline Query reference & Query & Remarks \\
\hline 1 & $\begin{array}{l}\text { AUTHOR: Please identify and encircle the forename and surname } \\
\text { of all authors. }\end{array}$ & \\
\hline 2 & AUTHOR: Please check the layout of Tables 1 -3. & $\begin{array}{l}\text { AUTHOR: Figure } 1 \text { was not cited in the text. An attempt has been } \\
\text { made to insert the figure into a relevant point in the text - please } \\
\text { check that this is OK. If not, please provide clear guidance on } \\
\text { where it should be cited in the text. }\end{array}$ \\
\hline 3 & $\begin{array}{l}\text { AUTHOR: Figure } 3 \text { has been saved at a low resolution of } 242 \text { dpi. } \\
\text { Please resupply at } 600 \text { dpi. Check required artwork specifications } \\
\text { at http://authorservices.wiley.com/bauthor/illustration.asp }\end{array}$ & \\
\hline 4 & $\begin{array}{l}\text { AUTHOR: Figure } 4 \text { has been saved at a low resolution of } 204 \text { dpi. } \\
\text { Please resupply at } 600 \text { dpi. Check required artwork specifications } \\
\text { at http://authorservices.wiley.com/bauthor/illustration.asp }\end{array}$ & \\
\hline 5 & $\begin{array}{l}\text { AUTHOR: Figure } 2 \text { is of poor quality. Please check required } \\
\text { artwork specifications at http://authorservices.wiley.com/bauthor/ } \\
\text { illustration.asp }\end{array}$ & \\
\hline 6 &
\end{tabular}

